\title{
Wide-Field Imaging Interferometry Spatial-Spectral Image Synthesis Algorithms
}

\author{
Richard G. Lyon, David T. Leisawitz, Stephen A. Rinehart, Nargess Memarsadeghi, \\ Evan J. Sinukoff \\ NASA/Goddard Space Flight Center, Greenbelt, MD 20771
}

\section{WORD ABSTRACT}

Developed is the algorithmic approach for wide field of view interferometric image synthesis. The collected data consists a set of image data cubes, one cube per baseline. These cubes are each 3D consisting of an array 2D detector counts versus delay line position. Moving the baseline and moving a delay line allows collection of a large set of interferograms over a $2 \mathrm{D}$ wide field detector grid; one interferogram per detector pixel per baseline. This aggregate set of interferograms, is algorithmically processed to construct a single spatial-spectral cube with angular resolution approaching the ratio of the wavelength to longest baseline.

\begin{abstract}
Developed is an algorithmic approach for wide field of view interferometric spatial-spectral image synthesis. The data collected from the interferometer consists of a set of double-Fourier image data cubes, one cube per baseline. These cubes are each three-dimensional consisting of arrays of two-dimensional detector counts versus delay line position. For each baseline a moving delay line allows collection of a large set of interferograms over the 2D wide field detector grid; one sampled interferogram per detector pixel per baseline. This aggregate set of interferograms, is algorithmically processed to construct a single spatial-spectral cube with angular resolution approaching the ratio of the wavelength to longest baseline. The wide field imaging is accomplished by insuring that the range of motion of the delay line encompasses the zero optical path difference fringe for each detector pixel in the desired field-of-view. Each baseline cube is incoherent relative to all other baseline cubes and thus has only phase information relative to itself. This lost phase information is recovered by having point, or otherwise known, sources within the field-ofview. The reference source phase is known and utilized as a constraint to recover the coherent phase relation between the baseline cubes and is key to the image synthesis. Described will be the mathematical formalism, with phase referencing and results will be shown using data collected from NASA/GSFC WideField Imaging Interferometry Testbed (WIIT).
\end{abstract}

Keywords: Interferometry, wide-field interferometry, double-Fourier, image synthesis, imaging interferometry

Biography: R. Lyon is an active researcher in techniques and instrumentation for exoplanet detection and characterization, wide field imaging interferometry, and precision wavefront and amplitude sensing and control. 